\title{
Decentralized Data-Driven Load Restoration in Coupled Transmission and Distribution System with Wind Power
}

Zhao, Jin ; Wu, Qiuwei; Hatziargyriou, Nikos D.; Li, Fangxing; Teng, Fei

Published in:

IEEE Transactions on Power Systems

Link to article, DOI:

10.1109/TPWRS.2021.3063114

Publication date:

2021

Document Version

Peer reviewed version

Link back to DTU Orbit

Citation (APA):

Zhao, J., Wu, Q., Hatziargyriou, N. D., Li, F., \& Teng, F. (2021). Decentralized Data-Driven Load Restoration in Coupled Transmission and Distribution System with Wind Power. IEEE Transactions on Power Systems, 36(5), 4435-4444. https://doi.org/10.1109/TPWRS.2021.3063114

\section{General rights}

Copyright and moral rights for the publications made accessible in the public portal are retained by the authors and/or other copyright owners and it is a condition of accessing publications that users recognise and abide by the legal requirements associated with these rights.

- Users may download and print one copy of any publication from the public portal for the purpose of private study or research.

- You may not further distribute the material or use it for any profit-making activity or commercial gain

- You may freely distribute the URL identifying the publication in the public portal 


\title{
Decentralized Data-Driven Load Restoration in Coupled Transmission and Distribution System with Wind Power
}

\author{
Jin Zhao, Student Member, IEEE, Qiuwei Wu, Senior Member, IEEE, Nikos. D. Hatziargyriou, Fel- \\ low, IEEE, Fangxing Li, Fellow, IEEE, Fei Teng, Member, IEEE
}

\begin{abstract}
This paper proposes a new decentralized datadriven load restoration (DDLR) scheme for transmission and distribution (TD) systems with high penetration of wind power. Robust DDLR models are constructed in order to handle uncertainties and ensure the feasibility of decentralized schemes. The Wasserstein metric is used to describe the ambiguity sets of probability distributions in order to build the complete DDLR model and realize computationally tractable formulation. A datadriven model-nested analytical target cascading (DATC) algorithm is developed to obtain the final load restoration result by iteratively solving small-scale mathematical models. The proposed DDLR scheme provides load restoration results with adjustable robustness, and performance efficiency is independent from the amount of data. The DDLR scheme makes full use of the available data while respecting information privacy requirements of independent operated systems, and ensures the feasibility of a decentralized load restoration strategy even in the worstcase condition. The effectiveness of the proposed method is validated using a small-scale TD system and a large-scale system with the IEEE 118-bus TS and thirty IEEE-33 DSs, showing high computational efficiency and superior restoration performance.
\end{abstract}

Index Terms-Data-driven optimization, distributionally robust, power system restoration, transmission and distribution system, wind power.

\section{INTRODUCTION}

$\mathrm{F}$ OLLOWING a complete or partial collapse of a power system, coordination among different entities is essential to complete the restoration process [1]. Conventionally, bulk system restoration follows a top down approach, i.e. it starts at the transmission level. The transmission system operator (TSO) leads the restoration process and coordinates facilities and load blocks in the transmission system (TS) without consideration of the distribution system (DS) conditions [2], [3], although the TS and DSs are physically interconnected. In modern grids however, two-way communication [4] between TSO and distribution system operator (DSO) can facilitate the restoration process using DS resources and avoid security violations by coordinating entities at the transmission level and distribution level.

This work was supported by, (Corresponding author: Qiuwei Wu).

J. Zhao and Q. Wu are with the Center for Electric Power and Energy, Department of Electrical Engineering, Technical University of Denmark, Kgs. Lyngby, DK 2800 (e-mail: hizhaojin@163.com; qw@elektro.dtu.dk)

N. D. Hatziargyriou is with the National Technical University of Athens, Athens 15773, Greece (e-mail: nh@power.ece.ntua.gr)

F. Li is with Department of EECS, The University of Tennessee, Knoxville. TN 37996, USA (e-mail: fli6@utk.edu)

F. Teng is the Electrical and Electronic Engineering Department, Imperial College London, London, SW7 2AZ, U.K. (e-mail: f.teng@imperial.ac.uk)
The TS and DSs are coupled, but they are independently operated by the TSO and DSOs [5], e.g. in the widespread August 2019 power outage event in the UK, the TSO was responsible for frequency regulation and DSOs made decisions about load shedding and load reconnection within their regions [6]. Accordingly, in order to maintain the independent decision-making of TSO and DSOs and avoid data processing burden, decentralized methods can be applied to realize the load restoration of coupled transmission and distribution systems (TDS). Based on decomposition and coordination, decentralized optimization models are built in [7] and [8] and the TDS restoration strategy is obtained through iterative calculation. Ensuring consistent TDS boundary variables is vital to obtaining a decentralized strategy [4], [7]-[10]. Moreover, the utilization of wind power for TDS restoration has been emphasized in different studies. The uncertain nature of wind power based sources (WPSs), however, affects seriously the consistency of boundary variables if not properly handled, which is guaranteed under deterministic conditions. Consequently, a deterministic decentralized strategy fails to coordinate effectively the TSO and DSOs and may even mislead the restoration process. Therefore, uncertainties need to be considered in the decentralized TDS load restoration with WPSs.

In the restoration process, stochastic programming (SP) [11], [12] and robust optimization (RO) [13]-[15] are widely used methods to handle uncertainties. The SP method generally relies on sampling approaches based on a certain probability distribution. However, with a limited amount of data, the actual distribution is hard to estimate with enough accuracy. The RO method considers the worst-case within the uncertain set and requires less information, however, the obtained restoration strategy may be very conservative. Although the budget of uncertainty can be used to alleviate conservativeness [16], probability distribution information of uncertainties is not regarded as making good use of available data in the restoration process. Retaining robustness and conservativeness mitigation characteristics, data-driven optimization can be applied to deal with uncertainties [17]-[20]. Based on a distributionally robust method, the data-driven optimization presents a mathematical framework that is well-suited to the limited information available in real-life problems and captures the decision maker's attitude toward uncertainty [21]. Therefore, considering data assisted power system operation, data-driven methods can be used in the decentralized load restoration scheme to dig potential information from limited data, and finally provide a robust yet less conservative result for TDS with wind power penetra- 
tion.

In this paper, a new decentralized data-driven load restoration (DDLR) scheme is proposed for the restoration of WPSs integrated TDS. First, the DDLR framework is established with compact models of the TSO and DSOs. Then, detailed DDLR models and Wasserstein metric based ambiguity sets are presented. Finally, the DDLR models are reformulated into computationally tractable forms, and a data-driven modelnested analytical target cascading (DATC) algorithm is applied to provide the DDLR strategy with flexible robustness.

This paper is the further work of [7]. Ref. [7] proposed the basic idea of a decentralized restoration scheme. It built the decentralized load restoration models and provided a deterministic decentralized load restoration strategy. This paper deals with uncertain conditions. The decentralized data-driven load restoration models are built, and the original decentralized algorithm is improved as the DATC in order to efficiently solve data-driven optimization models.

The contributions of this paper can be summarized as follows: 1) A new DDLR scheme is proposed to provide a robust TDS coordinated load restoration strategy considering uncertainties of wind power penetrated TDS load restoration. The feasibility of the decentralized load restoration scheme under uncertain conditions is ensured and the conservativeness of the robust strategy is alleviated. 2) The DDLR method makes full use of the available data respecting privacy requirements of independent system operators. In a decentralized way, the DDLR method maintains independent decision-making of the TSO and DSOs, which allows decentralized data processing in the large-scale TDS with tremendous data from uncertain sources. 3) The DATC algorithm is developed to provide the DDLR strategy. Therein, DDLR models are transformed into computation tractable small-scale quadratic programs (QP) and mixed integer quadratic programs (MIQP). The DATC algorithm uses a data-driven iterative calculation process for parallel solution of these models presenting high computational efficiency irrespective of the amount of data.

The rest of the paper is organized as follows: Section II provides the framework of DDLR with compact models. Detailed DDLR models as well as the application of the Wasserstein metric are presented in Section III. Section IV shows the model reformulation and iterative calculation process based on the DATC algorithm. Section V provides case study results and discussions, followed by the conclusions.

\section{FRAMEWORK OF DATA-DRIVEN LOAD RESTORATION IN TDS WITH WPSS INTEGRATION}

This section establishes the framework of the decentralized data-driven TDS load restoration. The structure of the TDS is presented, and the compact models of DDLR are designed to handle uncertainties.

\section{A. Coordinated load restoration of TDS}

The basic structure of the TDS [9], [10] is shown in Fig. 1. In the TS large thermal generators (TG) and large-scale WPSs are connected. DS systems have radial networks, and can be distinguished in active DSs (ADSs), formed by the connection of distributed generators (DGs) and loads and passive DSs (PDSs) that feed only loads. The TS and DS are physically connected through transformers at substations, and they are separately operated by the TSO and DSOs. The TD system coordinated restoration considers the detailed DS operation condition, which is the main difference from conventional restoration. In the restoration process, DSs can provide local power supply to the end-users, and they are allowed to feed power to the TS [4]. In a decentralized way, the coordinated TDS restoration speeds up load recovery by flexibly dispatching generation both in the TS and DSs [7], [8].

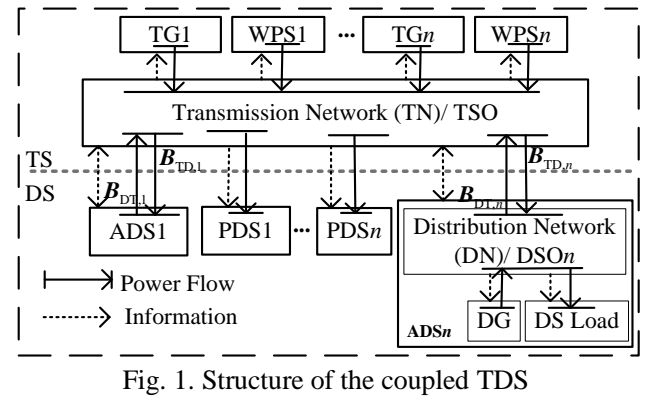

The key in decentralized load restoration is the decoupling of the TS and DS boundary variables between the TS and each DS. Under deterministic conditions, the Lagrangian decomposition method [22] is used to transform the boundary consistent constraints (1) into penalty functions $\Phi\left(\boldsymbol{\sigma}_{j}\right)$ and $\Phi(\boldsymbol{\sigma})$ added into the objective functions of the TSO model and each DSO model.

$$
\begin{gathered}
\boldsymbol{\sigma}_{j}=\boldsymbol{B}_{\mathrm{TD}, j}-\boldsymbol{B}_{\mathrm{DT}, j}=0 \quad \forall j \in \Omega_{\mathrm{Dis}} \\
\Phi\left(\boldsymbol{\sigma}_{j}\right)=\boldsymbol{v}_{j} \boldsymbol{\sigma}_{j}+\left\|\boldsymbol{w}_{j} \circ \boldsymbol{\sigma}_{j}\right\|_{2}^{2} \\
\Phi(\boldsymbol{\sigma})=\boldsymbol{v}^{\mathrm{T}}+\left\|\boldsymbol{w} \circ \boldsymbol{\sigma}^{\mathrm{T}}\right\|_{2}^{2}=\sum_{j \in \Omega_{\mathrm{Dis}}}\left(\boldsymbol{v}_{j} \boldsymbol{\sigma}_{j}+\left\|\boldsymbol{w}_{j} \circ \boldsymbol{\sigma}_{j}\right\|_{2}^{2}\right)
\end{gathered}
$$

where $\boldsymbol{B}_{\mathrm{TD}, j}$ and $\boldsymbol{B}_{\mathrm{DT}, j}$ represent vectors of boundary variables between the TS and DS $j$, and $\boldsymbol{v}$ and $\boldsymbol{w}$ are vectors of penalty multipliers of model decoupling. $\Omega_{\text {Dis }}$ is the set of the DSs. The symbol $\circ$ represents the Hadamard product, which is an entry-wise multiplication of two vectors.

Decoupling the centralized TDS load restoration model provides the decentralized load restoration models for the TSO and each DSO. The decentralized load restoration strategy is feasible as long as the boundary variables of the TS and DSs are consistent. However, the load amount, large-scale WPS output in the TS and renewable energy based DGs output in DSs are uncertain. Consequently, the boundary powers are uncertain and therefore, deterministic models are unable to ensure feasibility under uncertain conditions. Decentralized data-driven models of each DSO and the TSO are proposed here in order to provide a robust decentralized load restoration strategy with limited data from uncertain sources. In this paper, WP based DGs (WP-DGs) are mainly considered, however other type of DGs can be similarly modeled.

\section{B. Compact DDLR models considering uncertainties}

Considering uncertainties, boundary variables in $\boldsymbol{B}_{\mathrm{TD}, j}$ and $\boldsymbol{B}_{\mathrm{DT}, j}$ are defined as uncertain active powers $P_{\mathrm{TD}, j}$ and $P_{\mathrm{DT}, j}$ between the TS and DSs. The goal of TDS load restoration is 
to maximize the amount of load recovery. Inequality and equality security constraints for the TS are represented by $\boldsymbol{g}_{\mathrm{TS}}()$ and $\boldsymbol{h}_{\mathrm{TS}}()$, while constraints for DSs are $\boldsymbol{g}_{\mathrm{DS}, j}()$ and $\boldsymbol{h}_{\mathrm{DS}, j}()$.

The decentralized data-driven DSO model is formulated as (4). The first term of the objective function (4a) maximizes the load recovery amount in the corresponding DS, and the second term minimizes the expected value of penalty function under distribution $\mathbb{P}_{\mathrm{D}, j}^{*}$ which is the worst-case distribution over an ambiguity set $\mathcal{P}_{\mathrm{D}, j}$.

$$
\begin{gathered}
\min _{\boldsymbol{x}_{\mathrm{DL}, j}}-\sum_{i \in \Omega_{\mathrm{DL}, j}}\left(c_{\mathrm{DL}, j, i} E_{\mathrm{DL}, j, i} x_{\mathrm{DL}, j, i}\right)+\sup _{\mathbb{D}_{\mathrm{D}, j} \in \mathcal{P}_{\mathrm{D}, j}} \mathbb{E}_{\mathbb{P}_{\mathrm{D}, j}}\left\{\Phi\left(\sigma_{j}\right)\right\}(4 \mathrm{a}) \\
\text { s.t. } \quad \boldsymbol{g}_{\mathrm{DS}, j}\left(\boldsymbol{x}_{\mathrm{DL}, j}, \boldsymbol{p}_{\mathrm{DL}, j}, \boldsymbol{p}_{\mathrm{DG}, j}, P_{\mathrm{DT}, j}\right) \leq 0 \quad j \in \Omega_{\mathrm{Dis}} \\
\boldsymbol{h}_{\mathrm{DS}, j}\left(\boldsymbol{x}_{\mathrm{DL}, j}, \boldsymbol{p}_{\mathrm{DL}, j}, \boldsymbol{p}_{\mathrm{DG}, j}, P_{\mathrm{DT}, j}\right)=0 \quad j \in \Omega_{\mathrm{Dis}}
\end{gathered}
$$

$E_{\mathrm{DL}, j, l}, x_{\mathrm{DL}, j, i}$ and $c_{\mathrm{DL}, j, i}$ are the expected load amount, load pickup decision variable and the weighting coefficients of load $\boldsymbol{i}$ in $\mathrm{DS} j, \boldsymbol{x}_{\mathrm{DL}, j}$ and $\boldsymbol{p}_{\mathrm{DL}, j}$ are sets of load pickup decision variables and uncertain load amounts in DS $j, \boldsymbol{p}_{\mathrm{DG}, j}$ and $\boldsymbol{p}_{\mathrm{DL}, j}$ are sets of uncertain active outputs of WP-DGs and load amount in DS $j, P_{\mathrm{DT}, j}$ is the uncertain boundary power following probability distribution $\mathbb{P}_{\mathrm{D}, j}$, and $\mathcal{P}_{\mathrm{D}, j}$ is the corresponding ambiguity set. $\Omega_{\mathrm{DL}, j}$ is the load node set of DS $j$.

The decentralized data-driven TSO model (5) coordinates the whole system by minimizing all the mismatches of boundary powers considering TS security constraints. Since frequency security is of vital importance for the bulk system load restoration process [23]-[26], a data-driven chance constraint (5d) for frequency is added into the model to enhance frequency security under uncertain conditions.

$$
\begin{gathered}
\min _{\boldsymbol{p}_{\mathrm{G}}, \boldsymbol{P}_{\mathrm{TD}}} \Phi(\boldsymbol{\sigma}) \\
\text { s.t. } \quad \boldsymbol{g}_{\mathrm{TS}}\left(\boldsymbol{p}_{\mathrm{G}}, \boldsymbol{p}_{\mathrm{RE}}, \boldsymbol{P}_{\mathrm{TD}}\right) \leq 0 \\
\boldsymbol{h}_{\mathrm{TS}}\left(\boldsymbol{p}_{\mathrm{G}}, \boldsymbol{p}_{\mathrm{RE}}, \boldsymbol{P}_{\mathrm{TD}}\right)=0 \\
\inf _{\mathbb{P}_{\mathrm{T}} \in \mathcal{P}_{\mathrm{T}}} \mathbb{P}_{\mathrm{T}}\left\{f\left(\boldsymbol{p}_{\mathrm{RE}}, \boldsymbol{P}_{\mathrm{TD}}\right) \leq \Delta f_{\max }\right\} \geq 1-\rho
\end{gathered}
$$

where $\boldsymbol{p}_{\mathrm{G}}, \boldsymbol{p}_{\mathrm{RE}}$ and $\boldsymbol{P}_{\mathrm{TD}}=\left[P_{\mathrm{TD}, 1}, \ldots P_{\mathrm{TD}, j} \ldots\right]$ are sets of active power outputs of generators, uncertain active outputs of WPS and boundary power variables in the TS, $\rho$ represents a low probability for the data-driven chance constraint, $f()$ is the frequency calculation function, and $\Delta f_{\max }$ is the allowed largest frequency deviation value.

TS loads are not considered because loads are rarely connected to the TS directly. If needed, variables of TS loads can be decoupled from the TSO model and form decentralized data-driven models in parallel with DSO models. In this way, the TSO model (5) has no integer variables.

The DDLR model concentrates on ambiguity sets describing the possible probability distributions derived from historic or online data in the load restoration process. Robustness is guaranteed with regard to the worst-case probability distribution in the ambiguity set. Normally, more data leads to a smaller ambiguity set and a less conservative strategy [20]. The data-based explicit expression of ambiguity sets of probability distributions, computation tractable model reformulation and the iterative calculation will be discussed in later sections.

\section{Methodology of DDLR scheme}

As shown in Fig. 2, the methodology of the DDLR scheme consists of three parts. Firstly, the DDLR model of the TSO and DSOs are built with ambiguity sets to represent uncertainties. The Wasserstein metric is used to provide the data-based explicit expression of ambiguity sets of probability distributions. Secondly, using mathematical transformations, the Wasserstein metric based DDLR models are transformed into computation tractable QP and MIQP models. Finally, an iterative calculation process is implemented according to the DATC algorithm. The DDLR strategy is obtained as long as the iterative calculation converges.

The three parts corresponding to model building, processing and solution are introduced in detail in Sec. III, Sec. IV. A and Sec. IV. B, respectively.

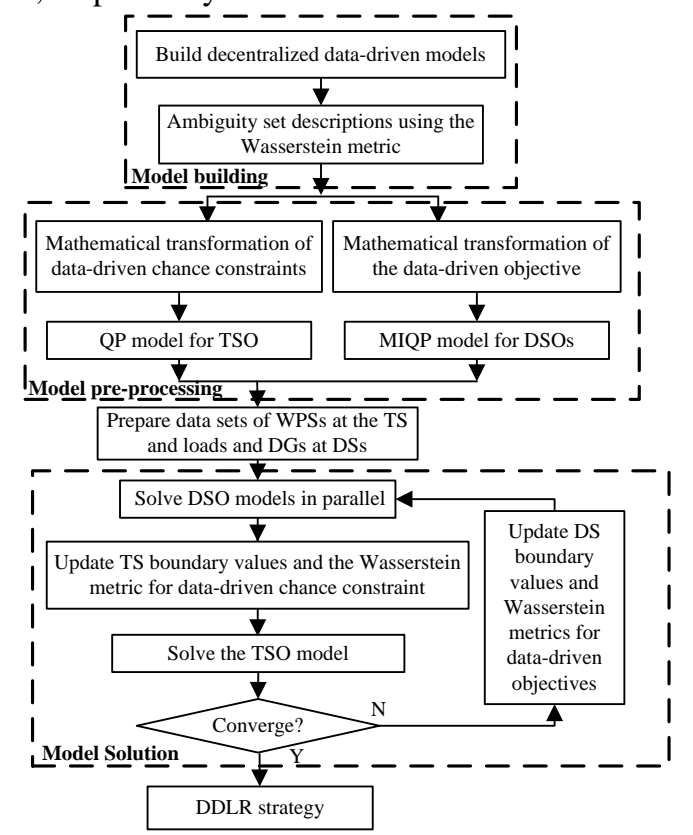

Fig. 2. Methodology of the DDLR scheme

\section{DDLR MODELS BASED ON WASSERSTEIN METRIC}

Detailed DDLR models that have the robustness to handle uncertainties are constructed in this section. First, the datadriven models for the TSO and DSOs are introduced. Then, a Wasserstein metric based expression of ambiguity sets is used to complete DDLR model building.

\section{A. Detailed DDLR models for TSO and DSOs}

The constraints for TSO and DSO DDLR models are all linearized. Detailed explanations about linearized TS AC power flow and linearized DS Disflow can be found in [7].

1) Detailed DDLR model for the TSO

The DDLR model (6)-(25) for the TSO is a frequency concerned chance-constrained optimization model corresponding to (5). The objective (6) is to coordinate the whole system by minimizing penalty functions of boundary variables between TS and DSs. Eq. (7)-(8) are generator output limits. Each restoration time step is constrained by (9). (10) and (11) determine the active and reactive power injections at each node of the TS. (12)-(18) are linearized AC power flow equations for 
the meshed transmission network. Branch power limits (19)(22) use square constraints to approximate the quadratic constraint of branch apparent power. Voltage and TDS boundary power limits are (23) and (24). Frequency limit (25) measures the largest frequency deviation in the transient process after system active power change. Specifically, the frequency constraint is in the data-driven chance-constrained form to enhance the uncertainty handling ability of the DDLR scheme.

$$
\begin{aligned}
& \min \sum_{j \in \Omega_{\mathrm{pis}_{\mathrm{is}}}}\left[v_{j}\left(P_{\mathrm{TD}, j}-P_{\mathrm{DT}, j}^{*}\right)+\left\|w_{j} \circ\left(P_{\mathrm{TD}, j}-P_{\mathrm{DT}, j}^{*}\right)\right\|_{2}^{2}\right] \\
& \max \left\{p_{\mathrm{G}, i}^{0}+r_{i} T, p_{\mathrm{G}, i, \min }\right\} \leq p_{\mathrm{G}, i} \leq \min \left\{p_{\mathrm{G}, i}^{0}+r_{i} T, p_{\mathrm{G}, i, \max }\right\} \\
& \forall i \in \Omega_{\mathrm{G}} \\
& p_{\mathrm{G}, i} \leq \sum_{i^{e} \in \Omega_{\mathrm{G}}, i^{e} \neq i}\left(r_{i^{i}} T_{\max }+p_{\mathrm{G}, i^{e}}\right)+\sum_{i \in \Omega_{\mathrm{RE}}} p_{\mathrm{RE}, i}-\sum_{i \in \Omega_{\mathrm{Qis}}} P_{\mathrm{TD}, i}-\sum_{i \in \Omega_{\mathrm{LL}}} P_{\mathrm{TLU}, i} \\
& T_{\text {min }} \leq T \leq T_{\text {max }} \\
& p_{n}=-P_{\mathrm{TD}, i}\left(i \in \Omega_{\mathrm{Dis}}\right)+p_{\mathrm{RE}, i}\left(i \in \Omega_{\mathrm{RE}}\right) \\
& +p_{\mathrm{G}, i}\left(i \in \Omega_{\mathrm{G}}\right)+0 \forall n \in \Omega_{\mathrm{TS}} \\
& q_{n}=q_{\mathrm{G}, i}\left(i \in \Omega_{\mathrm{G}}\right)+0 \forall n \in \Omega_{\mathrm{TS}} \\
& p_{n}=\sum_{\langle n, m\rangle \in \Omega_{\mathrm{R}}} P_{n m} \forall n \in \Omega_{\mathrm{TS}} \\
& q_{n}=\sum_{\langle n, m\rangle \in \Omega_{\mathrm{L}}} Q_{n m} \quad \forall n \in \Omega_{\mathrm{TS}} \\
& P_{n m}=g_{n m}-g_{n m} \operatorname{apr}\left(\cos \theta_{n m}\right)-b_{n m}\left(\theta_{n}-\theta_{m}\right) \\
& Q_{n m}=-b_{n m}-g_{n m}\left(\theta_{n}-\theta_{m}\right)+b_{n m} a p r\left(\cos \theta_{n m}\right) \\
& -b_{n m}\left(\delta_{n}-\delta_{m}\right) \\
& \operatorname{apr}\left(\cos \theta_{n m}\right) \geq \cos \theta_{\Delta}^{0} \\
& \operatorname{apr}\left(\cos \theta_{n m}\right) \leq-\sin \left(v d-\theta_{\Delta}^{0}\right)\left(\theta_{n}-\theta_{m}-v d+\theta_{\Delta}^{0}\right) \\
& +\cos \left(v d-\theta_{\Delta}^{0}\right) \quad v=1,2, \ldots, 2 h \\
& \left.V_{n}=1+\delta_{n} \quad \text { (Volts p.u. }\right) \\
& -S_{n m, \text { max }} \leq P_{n m} \leq S_{n m, \text { max }} \\
& -S_{n m, \text { max }} \leq Q_{n m} \leq S_{n m, \text { max }} \\
& -\sqrt{2} S_{n m, \text { max }} \leq P_{n m}+Q_{n m} \leq \sqrt{2} S_{n m, \text { max }} \\
& -\sqrt{2} S_{n m, \text { max }} \leq P_{n m}-Q_{n m} \leq \sqrt{2} S_{n m, \text { max }} \\
& V_{n, \text { min }}<V_{n}<V_{n, \text { max }} \quad \forall n \in \Omega_{\mathrm{TS}} \\
& -P_{\mathrm{TD}, j, \text { max }} \leq P_{\mathrm{TD}, j} \leq P_{\mathrm{TD}, j, \text { max }} \quad \forall j \in \Omega_{\mathrm{Dis}} \\
& \inf _{\mathbb{P}_{\mathrm{T}} \in \mathcal{P}_{\mathrm{T}}} \mathbb{P}_{\mathrm{T}}\left\{f\left(\boldsymbol{p}_{\mathrm{RE}}, \boldsymbol{P}_{\mathrm{TD}}\right) \leq \Delta f_{\max }\right\} \geq 1-\rho(25 a) \\
& f\left(\boldsymbol{p}_{\mathrm{RE}}, \boldsymbol{P}_{\mathrm{TD}}\right) \\
& =\left(\sum_{j \in \Omega_{\mathrm{Dis}}}\left(P_{\mathrm{TD}, j}-P_{\mathrm{TD}, j}^{0}\right)-\sum_{i \in \Omega_{\mathrm{RE}}}\left(p_{\mathrm{RE}, i}-p_{\mathrm{RE}, i}^{0}\right)\right) / \sum_{i^{i} \in \Omega_{\mathrm{i}, i}, i^{*} \neq i} \frac{\mathrm{s}_{i^{e}}}{\varepsilon_{i}}
\end{aligned}
$$

where $P_{\mathrm{TD}, j}$ and $P_{\mathrm{TD}}^{0}$ are boundary active power variables and the initial value for the TS between the TS and DS $j, P_{\mathrm{DT}_{j} j}^{*}$ is the value of boundary power transmitted from DSO $j, p_{\mathrm{G}, i}, p_{\mathrm{G}, i}^{0}$, $p_{\mathrm{G}, i, \min }, p_{\mathrm{G}, i, \max }$ and $r_{i}$ are respectively the active power output, initial active output, minimum and maximum active output, and ramping rate of generator $i$ in the TS, $T$ is the load pickup time at current step, $p_{\mathrm{RE}, i}$ and $p_{\mathrm{RE}, i}^{0}$ are the uncertain active power and initial power output of large-scale WPS $i$ in the TS, $q_{\mathrm{G}, i}$ is the reactive power of generator node $i, p_{n}$ and $q_{n}$ are the active and reactive power injection into node $n, P_{n m}, Q_{n m}$ and $S_{n m}$ are the active, reactive and apparent power flow on the TS line $n m, g_{n m}, b_{n m}$ and $\theta_{n m}$ are the conductance, susceptance and phase angle difference of the TS line $n m, \operatorname{apr}\left(\cos \theta_{n m}\right)$ is the approximate variable of $\cos \theta_{n m}, \theta_{\Delta}^{0}$ and $h$ are constants of phase angle difference and number of hyperplanes in the cosine polyhedron, $V_{n}$ and $\delta_{n}$ are voltage magnitude and its deviation variables of node $n, \mathrm{~s}_{i}$ and $\varepsilon_{i}$ are the capacity and frequency response rate of generator $i . \Omega_{\mathrm{G}}, \Omega_{\mathrm{RE}}$ and $\Omega_{\mathrm{TS}}$ are sets of generator nodes, WPS nodes and transmission lines in the TS.

2) Detailed DDLR model for DSO $j$

The DDLR model of DSO $j$ corresponding to (4) is shown in Eq. (26)-(38). The objective (26) is to maximize the load recovery in the related DS and minimize boundary mismatch under the worst-case probability distribution of boundary power. (27) is the power balance constraint. (28) and (29) determine the active and reactive power injections at each node of DS $j$. (30)-(32) are linearized DisFlow constraints for distribution radial networks. (33) is the voltage limit, (34)-(37) are branch power constraints, and (38) is the DS boundary power limit.

$$
\begin{aligned}
& \min _{x_{\mathrm{DL}, j}>x_{\mathrm{DL}, j}^{0}, j}-\sum_{i \in \Omega_{\mathrm{QL}, j}}\left(c_{\mathrm{DL}, j, i} E_{\mathrm{DL}, j, i} x_{\mathrm{DL}, j, i}\right) \\
& +\sup _{\mathbb{P}_{\mathrm{D}, j} \in \mathcal{P}_{\mathrm{D}, j}} \mathbb{E}_{\mathbb{P}_{\mathrm{D}, j}}\left\{v_{j}\left(P_{\mathrm{TD}, j}^{*}-P_{\mathrm{DT}, j}\right)+\left\|w_{j} \circ\left(P_{\mathrm{TD}, j}^{*}-P_{\mathrm{DT}, j}\right)\right\|_{2}^{2}\right\} \\
& P_{\mathrm{DT}, j}=\sum_{i \in \Omega_{\mathrm{DL}, j}} x_{\mathrm{DL}, i} p_{\mathrm{DL}, i}-\sum_{i \in \Omega_{\mathrm{DG}, j}} p_{\mathrm{DG}, i} \\
& p_{\mathrm{DS}, n}=-x_{\mathrm{DL}, i} E_{\mathrm{DL}, i}\left(i \in \Omega_{\mathrm{DL}, j}\right)+E_{\mathrm{DG}, i}\left(i \in \Omega_{\mathrm{DG}, j}\right) \\
& +\bar{P}_{\mathrm{DT}, j}\left(j \in \Omega_{\mathrm{Dis}}\right)+0 \quad \forall n \in \Omega_{\mathrm{DS}, j} \\
& q_{\mathrm{DS}, n}=-x_{\mathrm{DL}, i} q_{\mathrm{DL}, i}\left(i \in \Omega_{\mathrm{DL}, j}\right)+q_{\mathrm{DG}, i}\left(i \in \Omega_{\mathrm{DG}, j}\right)+0 \\
& \forall n \in \Omega_{\mathrm{DS}, j} \\
& P_{\mathrm{DS}, n_{\text {in }}}=P_{\mathrm{DS}, n_{\text {out }}}-p_{\mathrm{DS}, n_{\text {in }}}\left\langle n_{\text {in }}, n_{\text {out }}\right\rangle \in \Omega_{\mathrm{L}, \mathrm{DS} j} \\
& Q_{\mathrm{DS}, n_{\text {in }}}=Q_{\mathrm{DS}, n_{\text {out }}}-q_{\mathrm{DS}, n_{\text {in }}}\left\langle n_{\text {in }}, n_{\text {out }}\right\rangle \in \Omega_{\mathrm{L}, \mathrm{DS} j} \\
& V_{n_{\text {in }}}=V_{n_{\text {out }}}-\left(r_{n_{\text {out }}} P_{\mathrm{DS}, n_{\text {out }}}+x_{n_{\text {out }}} Q_{\mathrm{DS}, n_{\text {out }}}\right) / V_{0} \\
& V_{n, \text { min }}<V_{n}<V_{n, \text { max }} \quad \forall n \in \Omega_{\mathrm{DS}, j} \\
& -V_{0} \sqrt{L_{n, \text { max }} / r_{n}} \leq P_{\mathrm{DS}, n} \leq V_{0} \sqrt{L_{n, \text { max }} / r_{n}} \\
& -V_{0} \sqrt{L_{n, \text { max }} / r_{n}} \leq Q_{\mathrm{DS}, n} \leq V_{0} \sqrt{L_{n, \text { max }} / r_{n}} \\
& -V_{0} \sqrt{2 L_{n, \text { max }} / r_{n}} \leq P_{\mathrm{DS}, n}+Q_{\mathrm{DS}, n} \leq V_{0} \sqrt{2 L_{n, \text { max }} / r_{n}} \\
& -V_{0} \sqrt{2 L_{n, \text { max }} / r_{n}} \leq P_{\mathrm{DS}, n}-Q_{\mathrm{DS}, n} \leq V_{0} \sqrt{2 L_{n, \max } / r_{n}} \\
& -P_{\mathrm{DT}, j, \max } \leq P_{\mathrm{DT}, j} \leq P_{\mathrm{DT}, j, \max } \quad \forall n \in \Omega_{\mathrm{DS}, j}
\end{aligned}
$$

where $P_{\mathrm{DT}, j}, \bar{P}_{\mathrm{DT}, j}$ and $P_{\mathrm{TD}, j}^{*}$ are the boundary active power variable of DS $j$, sample mean of boundary active power of DS $j$ and the boundary power value transmitted from the TSO, $p_{\mathrm{DG}, i}$ and $E_{\mathrm{DG}, i}$ are the uncertain active power output and expected active power of DG $i, E_{\mathrm{DL}, i}$ denotes expected active power of DS load $i, q_{\mathrm{DL}, i}$ and $q_{\mathrm{DG}, i}$ are the reactive load amount and DG reactive power of node $i$ in DS $j, p_{\mathrm{DS}, n}$ and $q_{\mathrm{DS}, n}$ are the active and reactive power injection into DS node $n, n_{\text {in }}$ and $n_{\text {out }}$ are power injection/withdrawal nodes of a unidirectional 
single branch in the radial network, $P_{\mathrm{DS}, n}, Q_{\mathrm{DS}, n}$ and $L_{n, \max }$ are active, reactive power flow and apparent power limit on $n$th DS line, and $r_{n}$ and $b_{n}$ are the resistance and reactance of $n$th DS line. $\Omega_{\mathrm{DL}, j}, \Omega_{\mathrm{DG}, j}, \Omega_{\mathrm{DS}, j_{j}}$ and $\Omega_{\mathrm{L}, \mathrm{DS} j}$ are sets of loads, DGs, nodes and lines in DS $j$, respectively.

Instead of using a certain probability distribution or constructing a deterministic uncertainty set, the DDLR models carry all possible probability distributions in ambiguity sets which are data-driven and assumed to contain the true distribution. Therefore, the description of ambiguity sets is of vital importance to perform the DDLR method.

\section{B. Description of ambiguity set based on Wasserstein metric}

With finite samples $\left[p_{1}^{*}, p_{2}^{*}, \ldots p_{N}^{*}\right]$, the true distribution $\mathbb{P}$ of the uncertain variable $p$ is difficult to extract directly. Instead, the empirical distribution $\mathbb{P}_{N}=1 / N \sum_{k=1}^{N} \delta_{k}$ can be used as an estimation of the true distribution, where $\delta_{k}$ represents the $\mathrm{Di}$ rac measure on $p_{k}^{*}$. The 'distance' between the empirical distribution and the actual one can be represented by the Wasserstein metric. All distributions within the distance form an ambiguity set. Using the Wasserstein metric, the ambiguity set contains the actual distribution as much as possible, and it has the convergence attribute that $\mathbb{P}_{N}$ converges to $\mathbb{P}$ as $N \rightarrow \infty$ [20]. Moreover, it has a computationally tractable model reformulation.

For probability distributions $\mathbb{P}_{N}, \mathbb{P} \in \mathcal{P}(\Xi)$, the Wasserstein metric can be defined as (39) [18]:

$$
W\left(\mathbb{P}_{N}, \mathbb{P}\right)=\inf \left\{\int_{\Xi^{2}}\left\|p_{N}^{*}-p\right\| \Pi\left(d p_{N}^{*}, d p\right)\right\}
$$

where $\Pi$ is the joint distribution of $p_{\mathrm{N}}^{*}$ and $p$ with marginal distribution $\mathbb{P}_{\mathrm{N}}$ and $\mathbb{P},\|\|$ is used as $l_{1}$ norm \|\|$_{1}$ for its superior numerical tractability. Then, the ambiguity set (40) is defined as a Wasserstein ball centered at $\mathbb{P}_{\mathrm{N}}$ with radius $\gamma(N)$ [20]:

$$
\mathcal{P}:=\left\{\mathbb{P} \in \mathcal{R}(\Xi) \mid W\left(\mathbb{P}_{N}, \mathbb{P}\right) \leq \gamma(N)\right\}
$$

By adjusting the radius of the ambiguity set, the degree of conservatism of the optimization problem can be controlled. According to the characteristics of the ambiguity set, $\gamma(N)$ is a decreasing function to bound $W()$ as small as possible. The derivation of $\gamma(N)$ is based on statistical methods, and [19] provides one with good control of conservativeness.

$$
\gamma(N)=C \sqrt{1 / N \log (1 / 1-\beta)}
$$

The value of $C$ can be estimated from data based on (42).

$$
C \approx \inf _{\alpha>0} 2 \sqrt{1 / 2 \alpha\left(1+\ln \left(1 / N \sum_{k=1}^{N} e^{\alpha\left\|p_{k}^{*}-\mu^{*}\right\|_{1}^{2}}\right)\right)}
$$

where $\mu^{*}$ is the sample mean and the minimization over $\alpha$ can be done by the bisection search method.

With this method, (43) is satisfied. A Wasserstein ball with radius $\gamma(N)$ can thus be viewed as a confidence set for the unknown actual distribution, as in statistical testing.

$$
P\left(W\left(\mathbb{P}_{N}, \mathbb{P}\right) \leq \gamma\right) \geq 1-\exp \left(-N \gamma^{2} / C^{2}\right)
$$

\section{SOlution METHOD OF DECENTRALIZEd DATA-DRIVEN LOAD RESTORATION MODELS}

In order to obtain the DDLR strategy, the DDLR models need to be reformulated and be solved with computational tractable forms. First, data-driven chance constraints and the worst-case expected boundary penalty function are reformulated. Then, the DATC algorithm is applied to iteratively solve the reformulated DDLR models.

\section{A. Tractable transformation of DDLR models}

1) Reformulation of the data-driven TSO model

For the TSO model (6)-(25), the chance constrained frequency limit (25) is the only computationally intractable part. Set $p_{\mathrm{T}, \mathrm{RE}}$ as the summary of all the WPS production uncertain variables, and $\boldsymbol{x}$ denotes other decision variables in (25). The general form for (25) with linear function $f()$ is:

$$
\inf _{\mathbb{P}_{\mathrm{T}} \in \mathcal{P}_{\mathrm{T}}} \mathbb{P}_{\mathrm{T}}\left\{f\left(\boldsymbol{x}, p_{\mathrm{T}, \mathrm{RE}}\right) \leq \Delta f_{\max }\right\} \geq 1-\rho
$$

The data-driven chance constraint (44) makes it difficult to derive directly a tractable equivalent reformulation. Therefore, instead of performing mathematical transformation, a convex conservative approximation for (44) is used. The determined uncertain set $\Omega$ as well as the robust constraint (45) implies the chance constraint (44).

$$
f\left(\boldsymbol{x}, p_{\mathrm{T}, \mathrm{RE}}\right) \leq \Delta f_{\text {max }} \quad \forall p_{\mathrm{T}, \mathrm{RE}} \in \Omega
$$

The uncertain set $\Omega$ can be expressed as a data-driven hypercube [19]. The sample set $\left[p_{\mathrm{T}, \mathrm{RE}, 1}^{*}, p_{\mathrm{T}, \mathrm{RE}, 2, \ldots}^{*}, p_{\mathrm{T}, \mathrm{RE}, \mathrm{N}}^{*}\right]$ is standardized by (46) to compute the boundary of the hypercube.

$$
\omega_{k}^{*}=\Sigma^{-1 / 2}\left(p_{\mathrm{T}, \mathrm{RE}, k}^{*}-\mu\right) \quad k=1,2 \ldots, N
$$

where $\mu$ and $\Sigma$ are respectively the sample mean and covariance. $\omega$ is the standardized uncertain variable which has mean 0 , covariance $I$ and support $\Psi=\{-\zeta \leq \omega \leq \zeta\}$. Set $\mathbb{P}_{\mathrm{T}}^{\text {std }}$ and $\mathcal{P}_{\mathrm{T}}^{\omega}$ as the true distribution and ambiguity set of $\omega$. Then, an uncertain set is constructed in (47) which means that the probability of $\omega$ not belonging to $\Psi$ is not larger than $\rho$.

$$
\sup _{\mathbb{P}_{T}^{\text {sud }} \in \mathcal{P}_{\mathrm{T}}^{\text {sdd }}} \mathbb{P}_{\mathrm{T}}^{\text {std }}(\omega \notin \Psi) \leq \rho
$$

Accordingly, $\Omega=\Sigma^{1 / 2} \Psi+\mu$ can be the uncertain set in (45) that represents (44). Obviously, the determination of the boundary of $\Psi$ is critical to find the final uncertain set, and the boundary $\zeta$ needs to be minimized to reduce conservatism. Therefore, an optimization model (48) is built to acquire $\zeta$.

$$
\begin{aligned}
& \min _{0 \leq \zeta \leq \zeta_{\max }} \zeta \text { s.t. } \sup _{\mathbb{P}_{\mathrm{T}}^{\text {sd }} \in \mathcal{P}_{\mathrm{T}}^{\text {sd }}} \mathbb{P}_{\mathrm{T}}^{\text {std }}(\omega \notin \Psi) \leq \rho \\
& \min _{0 \leq \zeta \leq \zeta_{\max } \zeta} \text { s.t. } G\left(\kappa_{\mathrm{T}}, \zeta\right) \leq \rho, \kappa_{\mathrm{T}} \geq 0 \\
& G\left(\kappa_{\mathrm{T}}, \zeta\right)=\kappa_{\mathrm{T}} \gamma_{\mathrm{T}}+1 / N \sum_{k=1}^{N}\left(1-\kappa_{\mathrm{T}}\left(\zeta-\left\|\omega_{k}^{*}\right\|_{\infty}\right)^{+}\right)^{+}
\end{aligned}
$$

Using Lemma 2 in [27], (48) is equal to (49) which has a unique minimum. $G\left(\kappa_{\mathrm{T}}, \zeta\right)$ is non-smooth but monotonous w.r.t. $\zeta$ so that (49) can be solved by the nested bisection search method described in [27]. $(x)^{+}=\max (x, 0)$.

Finally, (44) can be replaced by (45) with $\Omega=\sum^{1 / 2} \Psi+\mu$ as long as the boundary $\zeta$ of the hypercube is obtained. The 
equality in (45) should be ensured under the uncertain set $\Omega$.

The reformulated DDLR model of the TSO is (50) which is a QP model with the quadratic objective and linear constraints.

$$
\begin{array}{ll}
\text { Obj : } & \text { (6) } \\
\text { s.t. } & \text { (7) }-(24),(25 \mathrm{~b}) \\
& p_{\mathrm{T}, \mathrm{RE}}=\sum_{i \in \Omega_{\mathrm{RE}}} p_{\mathrm{RE}, i} \\
& f\left(\boldsymbol{P}_{\mathrm{TLU}}, \boldsymbol{P}_{\mathrm{TD}}, p_{\mathrm{T}, \mathrm{RE}}\right) \leq \Delta f_{\max } \forall p_{\mathrm{T}, \mathrm{RE}} \in \Omega
\end{array}
$$

2) Reformulation of the data-driven DSO model

For the DSO model (26)-(38), the intractable parts are the worst-case expected penalty in the second stage. The penalty $\Phi\left(\sigma_{j}\right)$ for DS $j$ is a quadratic function of the following form:

$$
\Phi\left(x, P_{B}\right)=a P_{B}^{2}-b P_{B}+c
$$

where $P_{B}$ represents $P_{\mathrm{DT}, j}$ in (26), $x$ denotes other corresponding decision variables, and $a, b$, and $c$ are parameters of the quadratic function.

$$
\begin{aligned}
& \sup _{\mathbb{P}_{\mathrm{L}} \in \mathcal{P}_{\mathrm{L}}} \mathbb{E}_{\mathbb{P}_{\mathrm{L}}}\left\{\Phi\left(\boldsymbol{x}, P_{B}\right)\right\} \\
= & \begin{cases}\inf _{\kappa_{\mathrm{L}} \geq 0} & \kappa_{\mathrm{L}} \gamma_{\mathrm{L}}+1 / N \sum_{k=1}^{N} \tau_{k} \\
\text { s.t. } & \sup _{P_{B, \text {, in }} \leq P_{B} \leq P_{B, \text { max }}}\left(\Phi\left(\boldsymbol{x}, P_{B}\right)-\kappa_{\mathrm{L}}\left\|P_{B}-P_{B, k}^{*}\right\|\right) \leq \tau_{k} \forall k \leq N\end{cases}
\end{aligned}
$$

Applying the strong duality result for Wasserstein distancebased data-driven optimization [20], the equality (52) is satisfied with the sample set $\left[P_{B, 1}^{*}, P_{B, 2}^{*}, \ldots P_{B, N}^{*}\right]$ of the uncertain variable $P_{B}$ and its support $\left[P_{B, \min }, P_{B, \max }\right] . \tau_{k}$ is an auxiliary variable.

Since $\Phi\left(x, P_{B}\right)$ is convex, the optimal solution of the supremum in (52) must be obtained at the vertexes $P_{B, \min }, P_{B, \max }$ and $P_{B, k}^{*}$ for any fixed $\kappa_{\mathrm{L}}$. Therefore, the optimization model in (52) can be transformed into a deterministic formulation (53).

$$
\begin{aligned}
\inf _{\kappa_{\mathrm{L}} \geq 0} & \kappa_{\mathrm{L}} \gamma_{\mathrm{L}}+1 / N \sum_{k=1}^{N} \tau_{k} \\
\text { s.t. } & \Phi\left(\boldsymbol{x}, P_{B, \text { min }}\right)+\kappa_{\mathrm{L}}\left(P_{B, \text { min }}-P_{B, k}^{*}\right) \leq \tau_{k} \quad \forall k \leq N \\
& \Phi\left(\boldsymbol{x}, P_{B, \text { max }}\right)-\kappa_{\mathrm{L}}\left(P_{B, \text { max }}-P_{B, k}^{*}\right) \leq \tau_{k} \forall k \leq N \\
& \Phi\left(\boldsymbol{x}, P_{B, k}^{*}\right) \leq \tau_{k} \forall k \leq N
\end{aligned}
$$

Although (53) is computationally tractable, the number of constraints and auxiliary variables increases proportionally with sample size. To avoid the computation burden of facing a large amount of data, a close upper approximation of (53) is derived in (54) [27].

$$
\begin{gathered}
\inf _{\kappa_{\mathrm{L}} \in R} \kappa_{\mathrm{L}} \gamma_{\mathrm{L}}+1 / N \sum_{k=1}^{N} \Phi\left(\boldsymbol{x}, P_{B, k}^{*}\right) \\
\text { s.t. }-\Phi^{\prime}\left(\boldsymbol{x}, P_{B, \min }\right) \leq \kappa_{\mathrm{L}} \\
\Phi^{\prime}\left(\boldsymbol{x}, P_{B, \max }\right) \leq \kappa_{\mathrm{L}}
\end{gathered}
$$

where $\Phi^{\prime}\left(x, P_{B}\right)$ is the derivative of $\Phi\left(x, P_{B}\right)$. This transformation eliminates the effect of data size on the computation of data-driven DSO models. Moreover, the optimal results of (54) and (53) have a very small difference which can be diminished as the sample size $N$ increases [27]. Finally, the DDLR model of DSO $j$ is reformulated as (55) where $S_{\mathrm{D}, j}=\left\{p_{\mathrm{DS}, n}, q_{\mathrm{DS}, n}\right.$,

$$
\begin{aligned}
& \left.P_{\mathrm{DS}, n}, Q_{\mathrm{DS}, n}, V_{\mathrm{DS}, n} \forall n \in \mathrm{N}_{\mathrm{DS}, j}\right\} . \\
& \min _{x_{\mathrm{DL}, j} x_{\mathrm{DL}, j}^{0}, s_{\mathrm{D}, j}, r_{\mathrm{D}, j}}-\sum_{i \in \Omega_{\mathrm{DL}, j}}\left(c_{\mathrm{DL}, j, i} E_{\mathrm{DL}, j, i} x_{\mathrm{DL}, j, i}\right) \\
& +\kappa_{\mathrm{D}, j} \gamma_{\mathrm{D}, j}+\frac{1}{N} \sum_{k=1}^{N}\left\{v_{j}\left(P_{\mathrm{TD}, j}^{*}-P_{\mathrm{DT}, j, k}\right)+w_{j}^{2}\left(P_{\mathrm{TD}, j}^{*}-P_{\mathrm{DT}, j, k}\right)^{2}\right\} \\
& \text { s.t. }-\left(2 w_{j}^{2} P_{\mathrm{DT}, j, \min }^{*}-v_{j}-2 w_{j}^{2} P_{\mathrm{TD}, j}^{*}\right) \leq \kappa_{\mathrm{D}, j} \\
& 2 w_{j}^{2} P_{\mathrm{DT}, j, \text { max }}^{*}-v_{j}-2 w_{j}^{2} P_{\mathrm{TD}, j}^{*} \leq \kappa_{\mathrm{D}, j} \\
& P_{\mathrm{DT}, j, k}=\sum_{i \in \Omega_{\mathrm{DL}, j}} x_{\mathrm{DL}, i} p_{\mathrm{DL}, i, k}^{*}-\sum_{i \in \Omega_{\mathrm{QG}, j}} p_{\mathrm{DG}, i, k}^{*} \\
& \bar{P}_{\mathrm{DT}, j}=\frac{1}{N} \sum_{k=1}^{N} P_{\mathrm{DT}, j, k} \\
& \text { (27) }-(37)
\end{aligned}
$$

\section{B. Iterative calculation of DDLR models}

In order to solve the DDLR models, the Wasserstein metric based ambiguity set description, the tractable model reformulation and the ATC method [28] are incorporated to develop the DATC algorithm. The DATC algorithm performs a data-driven iterative calculation process, and the final DDLR strategy is obtained when the iterative calculation process converges.

Step 0: Set $L_{\text {atc }}=1$ and ATC convergence thresholds $\varepsilon=$ $0.1 \%$. Set initial values of boundary variables $P_{\mathrm{TD}, j}^{*}=P_{\mathrm{TD}, j}^{L_{\mathrm{se}}}$ and define initial values of penalty multipliers $v_{j}^{*}=v_{j}^{L_{n}}$ and $w_{j}^{*}=w_{j}^{L_{k}}$ in the DS model (55). Prepare sample set of WPS output $[\boldsymbol{p}$ ${ }_{\mathrm{RE}, 1}^{*}, \ldots, \boldsymbol{p}_{\mathrm{RE}, N_{\mathrm{R}} \mathrm{E}}^{*}$ in TS, and obtain $\zeta$ for the TSO model (50).

Step 1: Arbitrarily select feasible binary load pickup decisions in $\boldsymbol{x}_{\mathrm{DL}, j}^{1}$, and prepare sample $\left[\boldsymbol{p}_{\mathrm{DL}, j, 1}^{*}, \ldots, \boldsymbol{p}_{\mathrm{DL}, j, N_{\mathrm{sL}}}^{*}\right]$ and $[\boldsymbol{p}$ $\left.{ }_{\mathrm{DG}, j, 1}^{*}, \ldots, \boldsymbol{p}_{\left.\mathrm{DG}, j, N_{\mathrm{ss}}\right]}^{*}\right]$ for loads and DGs in each DS. Obtain $P_{\mathrm{DT}, j, \mathrm{~min}}=$ $P_{\mathrm{DT}, j \text { min }}^{1}, P_{\mathrm{DT}, j, \text { max }}=P_{\mathrm{DT}, j \text { max }}^{1}$ and $\gamma_{\mathrm{D}, j}=\gamma_{\mathrm{D}, j}^{1}$ using $C$ defined by (42).

Step 2: Solve the DS model (55) with $P_{\mathrm{TD}, j}^{*}, v_{j}^{*}$ and $w_{j}^{*}$, and fixed $P_{\mathrm{DT}, j, \text { min }}, P_{\mathrm{DT}, j, \max }$ and $\gamma_{\mathrm{D}, j}$ obtained by step 1 . Get the optimal result $\boldsymbol{x}_{\mathrm{DL}, j}^{2}=\boldsymbol{x}_{\mathrm{DL}, j}^{*}$, and obtain $\gamma_{\mathrm{D}, j}=\gamma_{\mathrm{D}, j}^{2}$. Here the result $\boldsymbol{x}_{\mathrm{DL}, j}^{2}$ is feasible for the DSO model (55), and holds good optimality because the change of $\boldsymbol{x}_{\mathrm{DL}, j}$ almost has no influence on the value of $\gamma_{\mathrm{D}, j}$.

Step 3: Obtain the sample set of DS boundary power $\left[p_{\mathrm{DT}, j, 1}^{*}\right.$, $\left.p_{\mathrm{DT}, j 2}^{*}, \ldots p_{\mathrm{DT}, N \mathrm{~N}}^{*}\right]$, then, find the boundary of the Wessestein metric based data-driven uncertain set $\Omega_{\mathrm{DS}, j}$ using (46)-(49). Find

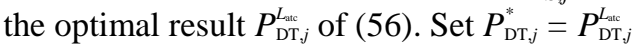

$$
\begin{array}{ll}
\max & v_{j}\left(P_{\mathrm{TD}, j}^{*}-P_{\mathrm{DT}, j}\right)+w_{j}^{2}\left(P_{\mathrm{TD}, j}^{*}-P_{\mathrm{DT}, j}\right)^{2} \\
\text { s.t. } & P_{\mathrm{DT}, j} \in \Omega_{\mathrm{DS}, j}
\end{array}
$$

Step 4: Collect $P_{\mathrm{DT}, j}^{*}$ from all the DSO models. Solve the TSO model (50) with $P_{\mathrm{DT}_{j}=}^{*}=P_{\mathrm{DT}, j}^{L_{\text {as }}}$ Obtain $P_{\mathrm{TD}, j}^{L_{\text {as }}+1}$.

Step 5: Check convergence by the consistency equation (57). If it is not satisfied and the iteration limit (58) is not reached, proceed to Step 6. Otherwise, stop and provide the final result.

$$
\begin{gathered}
\left|P_{\mathrm{TD}, j}^{L_{\text {atc }}+1}-P_{\mathrm{DT}, j}^{L_{\text {atc }}}\right| \leq \varepsilon \quad \forall j \in \Omega_{\mathrm{DS}} \\
L_{\mathrm{atc}} \leq L_{\max }
\end{gathered}
$$

Step 6: Update penalty parameters $v_{j}^{*}=v^{L_{w+1}+1}$ and $w_{j}^{*}=w^{L_{u x+1}}$ according to (59) and (60). Set $L_{\text {atc }}=L_{\text {atc }}+1$ and go to Step 1 .

$$
v_{j}^{L_{\text {atc }}+1}=v_{j}^{L_{\text {atc }}}+2\left(w_{j}^{L_{\text {atc }}}\right)^{2}\left(P_{\mathrm{TD}, j}^{L_{\text {atc }}+1}-P_{\mathrm{DT}, j}^{L_{\text {atc }}}\right) \quad \forall j \in N_{\mathrm{DS}}
$$




$$
w_{j}^{L_{\text {act }}+1}=\alpha w_{j}^{L_{\text {atc }}}(\alpha \geq 1) \quad \forall j \in N_{\mathrm{DS}}
$$

In this process, solving the TSO model (50) and DSO models (55) are QP and MIQP respectively with quadratic objective and linear constraints. The iterative calculation step 1-step 4 are performed in parallel for each DSO model. Therefore, although the TDS is a large scale system, calculations concern small-scale optimizations. Moreover, the DATC algorithm retains the strong convergence attribute of the ATC method [28]. Overall, the DATC algorithm-based data-driven iterative calculation is highly efficient with tractable model types, strong convergence and stable running time independent of data size.

\section{CASE STUdy}

Two test systems were used to validate the effectiveness of the proposed scheme, the T6D2 system and the T118D30 system, described next. WPS output and uncertain load amount respectively follow Weibull distribution and Gaussian distribution with $40 \%$ forecast error. Without special clarifications, the confidence levels are $\beta=95 \%$ and $\rho=5 \%$. The time interval of one restoration step is set as $5-10 \mathrm{~min}$. The weighting coefficients of loads are set as 10 in the T6D2 system and 100 in the T118D30 system. The initial values of boundary variables are set as 0 and initial values of penalty multipliers as set as 1 . All case studies were conducted using CPLEX V12.8.0 on a computer with Intel(R) Core(TM) i7$8550 \mathrm{U}$ CPU and 16 GB RAM.

\section{A. Computation efficiency of DDLR method}

The T6D2 test system consists of a 6 bus TS and two DSs with system data described in [7]. The G3 in the TS is replaced by uncertain WPSs. The uncertainty handling ability of DDLR optimization is compared to SP [11]-[12] and RO [14][15]. The SP-based decentralized models maximize load pickup amount and minimize the expected value of boundary mismatch penalty function with the uncertain variable following the empirical distribution from the sample set. It shares the same sample set with the DDLR method. Using the RO method, the decentralized models are in a two-stage robust form, i.e. maximize load recovery amount in the first stage and minimize the worst-case boundary mismatch with a max-min form in the second stage. The upper and lower bounds of the RO set are defined by the $40 \%$ forecast error.

Table I shows the results and calculation times of model solving in the first iteration. The RO focuses on the worst case in uncertain conditions and provides a conservative result with the lowest load recovery amount, $153 \mathrm{MW}$. Its calculation time is the largest because solving the two-stage RO requires extra iterative calculation based on the column-and-constraint generation method. SP gives the most optimistic result with the largest load recovery amount, $178 \mathrm{MW}$. Its model solving time grows with the data size. Compared to RO and SP, the DDLR method shows different load recovery results, while the solving time is always below $0.15 \mathrm{~s}$. When the data size is 600 , the DDLR method ensures out-of-sample performance by considering larger power deficiency beyond the data set, and allows less load recovery (168 MW) than the SP method (178 MW). Note that using the same sample sets, the probability distribution of the SP method is a special case included in the ambiguity set of the DDLR method. As more data is available, the ambiguity set shrinks and worst-case distribution approaches the true distribution. Consequently, the conservativeness of uncertainty handling is gradually reduced with improved load pickup amount from $168 \mathrm{MW}$ to $178 \mathrm{MW}$.

TABLE I

PERFORMANCE EFFICIENCY OF DIFFERENT METHODS

\begin{tabular}{ccccc}
\hline & Data size & DDLR & SP & RO \\
\hline Decentralized & 600 & 0.11 & 0.21 & \\
\cline { 2 - 4 } $\begin{array}{c}\text { model solving } \\
\text { time (s) }\end{array}$ & 3000 & 0.09 & 0.98 & \multirow{2}{*}{2.15} \\
\cline { 2 - 4 } $\begin{array}{c}\text { Recovery Load } \\
\text { (MW)/ Pickup }\end{array}$ & 6000 & 0.13 & 2.39 & \\
\cline { 2 - 4 } time (min) & 3000 & $168 / 8.56$ & $178 / 7.75$ & \\
\hline
\end{tabular}

In addition to the sample set, the robustness of the DDLR method is affected by the confidence level $\beta$ for the Wasserstein metric and the probability $\rho$ for chance constraints. Taking the WPS output (expected $50 \mathrm{MW}$ ) in the TS for example, Fig. 3 demonstrates the lower bound of the uncertain set derived from the Wasserstein metric based ambiguity set. Generally, the lower bound becomes lower with the increase of $\beta$ and decrease of $\rho$, and the change of lower bound value w.r.t $\rho$ is more obvious in an $\mathrm{x}-\mathrm{z}$ plane. The influence of $\beta$ is induced by Wasserstein metric radius $\gamma$. A larger $\beta$ leads to a larger $\gamma$ which means more possible distributions are contained in the ambiguity set. Thus, a more serious worst-case distribution is found leading to reduced WPS output. A smaller $\rho$ means the probability of uncertain WPS output not belonging to the reformulated uncertain set is lower, i.e. the bounds of uncertain set are wider. Therefore, smaller $\rho$ reduces the lower bound. A high confidence level, e.g. $\beta=95 \%$, is suggested to contain the worst-case distribution. The system operator can adjust probability $\rho$ according to the system restoration condition. With probability $\rho$ equal to $5 \%, 7 \%$ and $10 \%$, i.e. chanceconstraint levels $95 \%, 93 \%$ and $90 \%$, the load recovery amounts are 168MW 174MW and 183.5 MW, respectively. Under the same restoration condition, lower $\rho$ leads to higher reliability of results with less load recovery arrangement. This brings adjustable robustness for the uncertain load restoration process.

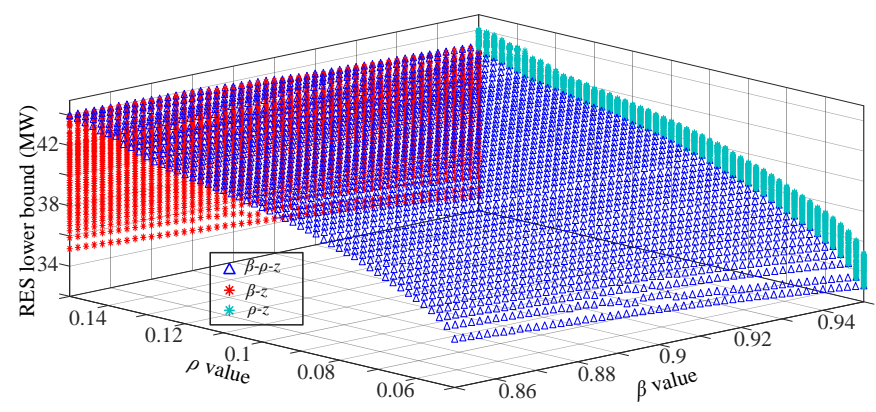

Fig. 3. Lower bound of WPS output with the change of $\beta$ and $\rho$

Overall, the DDLR method provides flexible uncertainty handling results based on available data and restoration conditions, and it has stable model solving time independent of the amount of data. 


\section{B. Load Restoration performance of DDLR scheme}

The T118D30 system consists of an IEEE 118-bus TS system and thirty IEEE 33-bus DSs with system data in [7]. At the TS level, ten wind farms are connected to buses $6,18,32$, 42, 56, 70, 77, 99, 113 and 116. In each DS, three WP-DGs are connected to buses 6,18 and 33 . The restoration performance of the proposed DDLR scheme is demonstrated comparing with the ones using a deterministic decentralized load restoration (ODLR) scheme [7] and centralized data-driven load restoration (CDLR) scheme. The CDLR scheme, using the data-driven model in [27], constructs a centralized TDS load restoration model with data-driven frequency-concerned chance constraint.

As Table II shows, all the schemes complete the load restoration process in three steps within $30 \mathrm{~min}$. Note that the CDLR scheme is presented to compare load restoration efficiency with the proposed DDLR method, however it may not be applicable in real TDSs, because detailed system information of DSOs is not normally available to the TSO. With different load recovery amounts, the strategy based on the DDLR scheme is different from the CDLR one. Although the DDLR's first step restores $50.60 \%$ load, while CDLR gets $51.76 \%$, this $1.16 \%$ difference is well acceptable. Apart from the small differences in the amount of load recovery and pickup time, the restoration efficiency of DDLR and CDLR are similar in later steps.

TABLE II

\begin{tabular}{cc|ccc}
\multicolumn{3}{c}{ RESTORATION EFFICIENCY COMPARISON OF DIFFERENT METHODS } \\
\hline Steps & & S1 & S2 & S3 \\
\hline \multirow{2}{*}{$\begin{array}{c}\text { Load pickup } \\
\text { time (min) }\end{array}$} & DDLR & 10 & 10 & 9.94 \\
\cline { 2 - 5 } & ODLR & 10 & 10 & 9.87 \\
\cline { 2 - 5 } & CDLR & 10 & 10 & 10 \\
\hline \multirow{2}{*}{$\begin{array}{c}\text { Load recovery } \\
(\%)\end{array}$} & DDLR & 50.60 & 86.60 & 100 \\
\cline { 2 - 5 } & ODLR & 59.51 & 94.22 & 100 \\
\cline { 2 - 5 } & CDLR & 51.76 & 85.20 & 100 \\
\hline \multirow{2}{*}{$\begin{array}{c}\text { Calculation time } \\
(\mathbf{s})\end{array}$} & DDLR & 30.87 & 3.55 & 0.68 \\
\hline \multirow{2}{*}{$\begin{array}{c}\text { Max mismatch } \\
(\boldsymbol{\%})\end{array}$} & ODLR & 10.11 & 3.75 & 0.64 \\
\cline { 2 - 5 } & DDLR & 0.00 & 0.00 & 0.00 \\
\hline
\end{tabular}

In a decentralized way, both DDLR and ODLR schemes need to perform iterative calculations, as shown in Fig. 4. The ODLR scheme shows the most optimistic strategy with $86.6 \%$ and $94.22 \%$ load recovery in S1 and S2. However, although the ODLR scheme obtains convergent results in Fig. 4 (a), the boundary mismatch in Fig. 6 (b) accumulates, as the restoration step proceeds. With boundary mismatch from 81.54 MW to $163.38 \mathrm{MW}$, the ODLR scheme is infeasible under uncertain conditions. The DDLR scheme ensures boundary consistency in the worst-case distribution as shown in Fig.4 (b), which guarantees the feasibility of the decentralized strategy under uncertain conditions. The proposed DDLR scheme finds the three step DDLR strategy through fast calculation. With the data-driven technique, the DDLR scheme uses a maximum $1.47 \mathrm{~s}$ for one iteration calculation. Without the data-driven technique, the average time for one iteration calculation of the ODLR scheme is $1.44 \mathrm{~s}$. Therefore, compared with ODLR, DDLR needs very little extra time to process data and handle uncertain conditions. This makes the proposed DDLR scheme suitable to support on-line restoration decision-making under uncertain conditions.
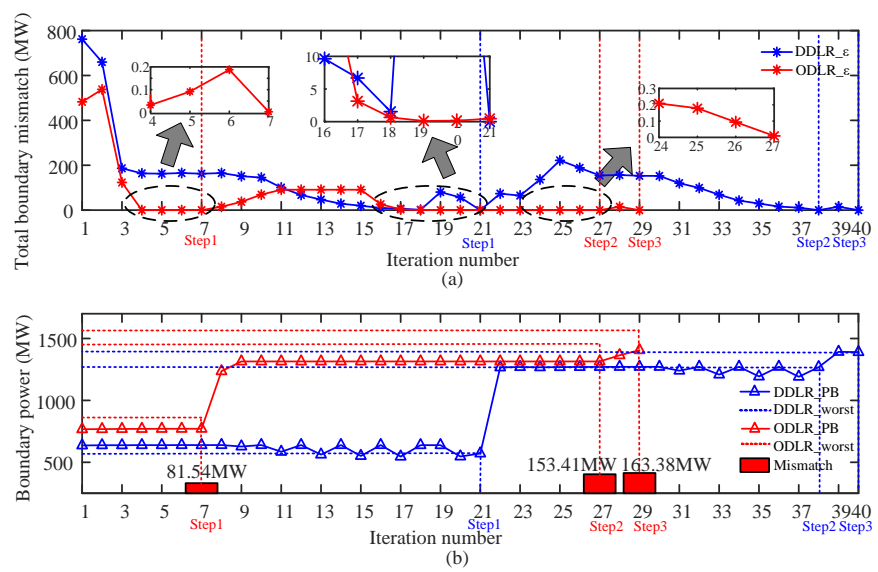

Fig. 4. Coverage process (a) and boundary conditions (b) of DDLR and ODLR in the load restoration process.

\section{CONCLUSION}

This paper presents a new DDLR scheme for TDS load restoration under uncertain conditions. The DDLR models of the TSO and DSOs are constructed with enough robustness to deal with worst-case distributions of boundary mismatch powers. Tractable computation is realized by the Wasserstein metric based ambiguity set description and DDLR model reformulations. Applying the developed DATC algorithm, the DDLR strategy can be obtained by iteratively solving small-scale QP and parallel MIQP models. Without bringing any computation burdens, the proposed method provides data-based results with flexible robustness to handle uncertain conditions. Moreover, it allows decentralized data processing under independent decision-making of the TSO and DSOs, and coordinates the whole TDS restoration by boundary information exchange. The case study results show the computation benefits and good load restoration performance of the proposed method. The DDLR method offers adjustable robustness as well as stable model-solving time independent of data size, and the corresponding DDLR scheme retains fast calculation characteristic meanwhile ensuring the feasibility of the decentralized TDS load restoration strategy under uncertain conditions.

With the concept of data-driven restoration strategies, future work can be focused on other restoration problems such as generator restart and parallel sectionalized restoration. Since the operation of modern power systems heavily relies on data, data-driven methods, model based or model free ones, are promising to dig potential information to benefit the whole restoration process.

\section{REFERENCES}

[1] M. M. Adibi and L. H. Fink, "Overcoming restoration challenges associated with major power system disturbances-restoration from cascading failures," IEEE Power Energy Mag., vol., no. 5, pp. 68-77, 2006.

[2] N.A.Fountas, N.D. Hatziargyriou, C. Orfanogiannis and A. Tasoulis, "Interactive long-term simulation for power system restoration planning," IEEE Trans. Power Syst., vol, 12 no. 1, pp. 61-68, 1997.

[3] F. Qiu and P. Li, "An integrated approach for power system restoration planning," Proc. IEEE, vol. 105, no. 7, pp. 1234-1252, 2017. 
[4] C. Chen, J. Wang, and D. Ton, "Modernizing distribution system restoration to active grid resiliency against extreme weather events: an integration solution," Proc. IEEE, vol.105, no. 7, pp.1267-1288, 2017.

[5] Z. Li, Q. Guo, H. Sun, and J. Wang, "Coordinated transmission and distribution ac optimal power flow," IEEE Trans. Smart Grid, vol. 9, no. 2, pp. 1228-1240, 2018.

[6] ESO final technical report: Technical report on the events of 9 August 2019, 2019, [Online]. Available: https://www.nationalgrideso.com/document/152346/download

[7] J. Zhao, H. Wang, Y. Liu, Q. Wu, Z. Wang and Y. Liu, "Coordinated restoration of transmission and distribution system using decentralized scheme," IEEE Trans. Power Syst., vol. 34, no. 5, pp. 3428-3442, 2019.

[8] R. Roofegari nejad, W. Sun and A. Golshani, "Distributed restoration for integrated transmission and distribution systems with DERs," IEEE Trans. Power Syst., vol. 34, no. 6, pp. 4964-4973, 2019.

[9] Z. Li, Q. Guo, H. Sun, and J. Wang, "Coordinated economic dispatch of coupled transmission and distribution systems using heterogeneous decomposition," IEEE Trans. Power Syst., vol. 31, no. 6, pp. 48171830, 2016.

[10] A. Kargarian and Y. Fu, "System of systems based security constrained unit commitment incorporating active distribution grids," IEEE Trans. Power Syst., vol. 29, no. 5, pp. 2489-2498, 2014.

[11] S. Lei, J. Wang, C. Chen, and Y. Hou, "Mobile emergency generator prepositioning and real-time allocation for resilient response to natural disasters," IEEE Trans. Smart Grid, vol. 9, no. 3, pp. 2030-2041, 2018.

[12] A. Golshani, W. Sun, Q. Zhou, Q. P. Zheng and Y. Hou, "Incorporating wind energy in power system restoration planning," IEEE Trans. Smart Grid, vol. 10, no. 1, pp. 16-28, 2019.

[13] K. Chen, W. Wu, B. Zhang and H. Sun, "Robust restoration decisionmaking model for distribution networks based on information gap decision theory," IEEE Trans. Smart Grid, vol. 6, no. 2, pp. 587-597, 2015.

[14] A. Golshani, W. Sun, Q. Zhou, Q. P. Zheng, J. Wong and F. Qiu, "Coordination of wind farm and pumped-storage hydro for a self-healing power grid," IEEE Trans. Sustain. energy, vol. 9, no. 4, pp. 1910-1920, 2018.

[15] J. Zhao, H. Wang, Y. Hou, Q. Wu, N. D. Hatziargyriou, Z. Wang and Y. Liu, "Robust distributed coordination of parallel restored subsystems in wind power penetrated transmission system," IEEE Trans. Power Syst., early access, 2020.

[16] S. Lei, Y. Hou, F. Qiu and J. Yan, "Identification of critical switches for integrating renewable distributed generation by dynamic network reconfiguration," IEEE Trans. Sustain. energy, vol. 9, no. 1, pp. 420-432, 2018.

[17] Y. Zhang, X. Ai, J. Fang, J. Wen, and H. He, "Data-adaptive robust optimization method for the economic dispatch of active distribution networks," IEEE Trans. Smart Grid, vol. 10, no. 4, pp. 3791-3800, 2019.

[18] P. M. Esfahani and D. Kuhn, "Data-driven distributionally robust optimization using the Wasserstein metric: Performance guarantees and tractable reformulations," Math. Program., vol. 24, no. 9, pp. 1-52, 2015.

[19] R. Zhu, H. Wei and X. Bai, "Wasserstein metric based distributionally robust approximate framework for unit commitment," IEEE Trans. Power Syst, vol. 34, no. 4, pp. 2991-3001, 2019.

[20] R. Gao and A. J. Kleywegt, "Distributionally robust stochastic optimization with Wasserstein distance" 2016, arXiv:1604.02199v2.

[21] D. Bertsimas and A. Thiele, "Robust and data-driven optimization: Modern decision-making under uncertainty," Tutorials in Operations Research: Models, Methods, and Applications for Innovative Decision Making, INFORMS, 2006.

[22] G. Monique and S. Kim, "Lagrangean decomposition: A model yielding stronger Lagrangean bounds," Math. Program., vol. 39, no. 2, pp. 215228, 1987.

[23] D. R. Medina, E. Rappold, O. Sanchez, et al. "Fast assessment of frequency response of clod load pickup in power system restoration," IEEE Trans. Power Syst, vol. 31, no. 4, pp. 3249-3256, 2016.

[24] W. Liu, Z, Lin, F. Wen, et al. "A wide area monitoring system based load restoration method." IEEE Trans. Power Syst, vol. 28, no.2, pp. 2025-2034, 2013.

[25] Chunyu Chen, Mingjian Cui, Xin Fang, Bixing Ren, and Yang Chen, "Load altering attack-tolerant defense strategy for secondary frequency control system", Appli. Energy, vol. 280, pp. 116015, 2020.

[26] Shuai Fan, Guangyu He, Xinyang Zhou, and Mingjian Cui, "Online optimization for networked distributed energy resources with timecoupling constraints", IEEE Trans. on Smart Grid, vol. 12, no. 1, pp. 251-267, 2021.
[27] C. Duan, W. Fang, L. Jiang, L. Yao, and L. Jun, "Distributionally robust chance-constrained approximate AC-OPF with Wasserstein metric," IEEE Trans. Power Syst., vol. 33, no. 5, pp. 4924-4936, 2018.

[28] N. F. Michelena, H. Park, and P. Y. Papalambros, "Convergence properties of analytical target cascading," AIAA J., vol. 41, no. 5, pp. 897-905, 2003.

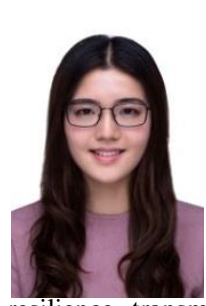

Jin Zhao (Member, IEEE) received the B.E. and Ph.D. degrees from Shandong University, Jinan, China, all in the electrical engineering, in 2015 and 2020, respectively.

She is currently a postdoctoral researcher at The University of Tennessee (UTK), USA. She was a research assistant at The University of Hong Kong (HKU), China. She was also a visiting $\mathrm{Ph} . \mathrm{D}$. student and postdoctoral researcher at Technical University of Denmark (DTU), Denmark. Her research interests include power system resilience, transmission \& distribution system restoration, renewable energy integration, power system optimization, deep learning and reinforcement learning.

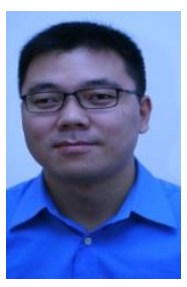

Qiuwei Wu (Senior Member, IEEE) obtained the PhD degree in Power System Engineering from Nanyang Technological University, Singapore, in 2009.

He was a senior R\&D engineer with VESTAS Technology R\&D Singapore Pte Ltd from Mar. 2008 to Oct. 2009 He has been working at Department of Electrical Engineering, Technical University of Denmark (DTU) since Nov. 2009 (PostDoc Nov. 2009-Oct. 2010, Assistant Professor Nov. 2010-Aug. 2013, Associate Professor since Sept. 2013). He was a visiting scholar at Department of Industrial Engineering \& Operations Research (IEOR), University of California, Berkeley, from Feb. 2012 to May 2012 funded by the Danish Agency for Science, Technology and Innovation (DASTI), Denmark. He was a visiting professor named by Y. Xue, an Academician of Chinese Academy of Engineering, at Shandong University, China, from Nov. 2015 to Oct. 2017. He was a visiting scholar at the Harvard China Project, School of Engineering and Applied Sciences, Harvard University from Nov. 2017 - Oct. 2018 funded by the Otto Mønsted Fond. His research interests are operation and control of power systems with high penetration of renewables, including wind power modelling and control, active distribution networks, and operation of integrated energy systems. He is an Editor of IEEE Transactions on Smart Grid and IEEE Power Engineering Letters. He is also an Associate Editor of International Journal of Electrical Power and Energy Systems, and Journal of Modern Power Systems and Clean Energy. $\mathrm{He}$ is a subject editor for IET Generation, Transmission \& Distribution, and IET Renewable Power Generation.

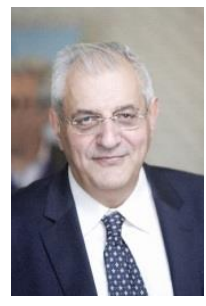

Nikos D. Hatziargyriou (Life Fellow, IEEE) is currently a Professor in Power Systems with the National Technical University of Athens. He has over ten years of industrial experience as the Chairman and CEO of the Hellenic Distribution Network Operator and as the Executive Vice-Chair of the Public Power Corporation. He was the Chair and currently the Vice-Chair of the EU Technology and Innovation Platform on Smart Networks for Energy Transition (ETIP-SNET) representing E.DSO. He has participated in more than 60 RD\&D projects funded by the EU Commission, electric utilities and manufacturers for both fundamental research and practical applications. He is author of the book, Microgrids: Architectures and Control and of more than 250 journal publications and 500 conference proceedings papers. He is included in the 2016, 2017, and 2019 Thomson Reuters lists of the top 1\% most cited researchers and he is Globe Energy Prize laureate 2020. He is an Honorary Member of CIGRE and the past Chair of CIGRE SC C6 "Distribution Systems and Distributed Generation". He is the past Chair of the Power System Dynamic Performance Committee (PSDPC) and currently the Editor-in-Chief of the IEEE Transactions on Power Systems. 


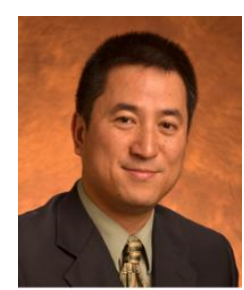

Fangxing Li (Fellow, IEEE) is also known as Fran Li. $\mathrm{He}$ received the B.S.E.E. and M.S.E.E. degrees from Southeast University, Nanjing, China, in 1994 and 1997, respectively, and the Ph.D. degree from Virginia Tech, Blacksburg, VA, USA, in 2001. Currently, he is the James W. McConnell Professor in electrical engineering and the Campus Director of CURENT at the University of Tennessee, Knoxville, TN, USA.

His current research interests include renewable energy integration, demand response, distributed generation and microgrid, energy markets, and power system computing. Since 2020, Prof. Li has been serving as the Editor-In-Chief of IEEE Open Access Journal of Power and Energy (OAJPE) and the Chair of IEEE PES Power System Operation, Planning and Economics (PSOPE) Committee. He has received a number of awards and honors including IEEE Fellow (Class of 2017), a R\&D 100 Award in 2020, a R\&D 100 Finalist in 2020, IEEE PES Distinguished Service Award in 2016, IEEE PES Technical Committee of the Year award in 2018, IEEE PES Technical Committee Prize Paper award in 2019, 3 best paper awards at international journals, and 6 best papers/posters at international conferences.

Fei Teng (Member, IEEE) received the B.Eng degree in Electrical Engineering and Automation from Beihang University, China, in 2009, and the $\mathrm{Ph} . \mathrm{D}$. degree in Electrical Engineering from Imperial College London, U.K., in 2015. Currently, he is a lecturer in the Department of Electrical and Electronic Engineering, Imperial College London, U.K. His research focuses on the efficient and resilient operation of the future cyber-physical power system. 